\title{
Reproducing Kernel Algorithm for the Analytical-Numerical Solutions of Nonlinear Systems of Singular Periodic Boundary Value Problems
}

\author{
Omar Abu Arqub \\ Department of Mathematics, Al-Balqa Applied University, Salt 19117, Jordan \\ Correspondence should be addressed to Omar Abu Arqub; o.abuarqub@bau.edu.jo
}

Received 8 December 2014; Accepted 2 May 2015

Academic Editor: Francesco Tornabene

Copyright (c) 2015 Omar Abu Arqub. This is an open access article distributed under the Creative Commons Attribution License, which permits unrestricted use, distribution, and reproduction in any medium, provided the original work is properly cited.

The reproducing kernel algorithm is described in order to obtain the efficient analytical-numerical solutions to nonlinear systems of two point, second-order periodic boundary value problems with finitely many singularities. The analytical-numerical solutions are obtained in the form of an infinite convergent series for appropriate periodic boundary conditions in the space $W_{2}^{3}[0,1]$, whilst two smooth reproducing kernel functions are used throughout the evolution of the algorithm to obtain the required nodal values. An efficient computational algorithm is provided to guarantee the procedure and to confirm the performance of the proposed approach. The main characteristic feature of the utilized algorithm is that the global approximation can be established on the whole solution domain, in contrast with other numerical methods like onestep and multistep methods, and the convergence is uniform. Two numerical experiments are carried out to verify the mathematical results, whereas the theoretical statements for the solutions are supported by the results of numerical experiments. Our results reveal that the present algorithm is a very effective and straightforward way of formulating the analytical-numerical solutions for such nonlinear periodic singular systems.

\section{Introduction}

Mathematical models of classical applications from physics, chemistry, and mechanics take the form of systems of singular periodic boundary value problems (BVPs) of second order which are a combination of singular differential system and periodic boundary conditions. Commonly, the singularity typically occurring at endpoints or in the form of a set of finite cardinality of the interval of integration. Periodic BVPs for systems of ordinary differential equations with singularities appear also in numerous applications which are of interest in modern applied mathematics. To name but a few, computations of self-similar blow-up solutions of nonlinear partial differential equations lead to the computation of problems from this class $[1,2]$, the density profile equation in hydrodynamics may be reduced to a system of singular periodic BVP $[3,4]$, the investigation of problems in the theory of shallow membrane caps is associated with such systems [5], and in ecology, in the computation of avalanche run-up, this problem class is translated into a system of singular periodic BVP $[6,7]$.

Most scientific problems and phenomenons in different fields of sciences and engineering occur nonlinearly. To set the scene, we know that except a limited number of these problems and phenomenons, most of them do not have analytical solutions. So these nonlinear equations should be solved using numerical methods or other analytical methods. Anyhow, when applied to the systems of singular periodic BVPs, standard numerical methods designed for regular BVPs suffer from a loss of accuracy or may even fail to converge [8-10], because of the singularity, whilst analytical methods commonly used to solve nonlinear differential equations are very restricted and numerical techniques involving discretization of the variables on the other hand give rise to rounding off errors. As a result, there are some restrictions to solve these singular periodic systems; firstly, we encountered with the nonlinearity of systems; secondly, these systems are singular BVPs with periodic boundary values. 
Investigation about systems of singular periodic BVPs numerically is scarce and missing. In this study, the reproducing kernel Hilbert space (RKHS) method has been successfully applied as a numerical solver for such systems. The present technique has the following characteristics; first, it is of global nature in terms of the solution obtained as well as its ability to solve other mathematical and engineering problems; second, it is accurate, needs less effort to achieve the results, and is developed especially for nonlinear cases; third, in the proposed technique, it is possible to pick any point in the given domain and as well the numerical solutions and their derivatives will be applicable; fourth, the approach does not require discretization of the variables, it is not effected by computation round off errors, and one is not faced with necessity of large computer memory and time; fifth, the proposed approach does not resort to more advanced mathematical tools; that is, the algorithm is simple to understand, implement, and should be thus easily accepted in the mathematical and engineering application's fields. More precisely, we provide the analytical-numerical solutions for the following differential singular system:

$$
\begin{aligned}
& u_{1}^{\prime \prime}(x)+\frac{a_{1}(x)}{p_{1}(x)} u_{1}^{\prime}(x)+\frac{b_{1}(x)}{q_{1}(x)} u_{1}(x) \\
& =f_{1}\left(x, u_{1}(x), u_{2}(x)\right), \\
& u_{2}^{\prime \prime}(x)+\frac{a_{2}(x)}{p_{2}(x)} u_{2}^{\prime}(x)+\frac{b_{2}(x)}{q_{2}(x)} u_{2}(x) \\
& =f_{2}\left(x, u_{1}(x), u_{2}(x)\right),
\end{aligned}
$$

subject to the following periodic boundary conditions:

$$
\begin{aligned}
& u_{1}(0)=u_{1}(1), \\
& u_{2}(0)=u_{2}(1), \\
& u_{1}^{\prime}(0)=u_{1}^{\prime}(1), \\
& u_{2}^{\prime}(0)=u_{2}^{\prime}(1),
\end{aligned}
$$

where $x \in(0,1), u_{s} \in W_{2}^{3}[0,1]$ are unknown functions to be determined, $f_{s}\left(x, v_{1}, v_{2}\right)$ are continuous terms in $W_{2}^{1}[0,1]$ as $v_{s} \in W_{2}^{3}[0,1], 0 \leq x \leq 1,-\infty<v_{s}<\infty$, and are depending on the system discussed, and $W_{2}^{1}[0,1], W_{2}^{3}[0,1]$ are two reproducing kernel spaces. Here, the two functions $p_{s}(x), q_{s}(x)$ may take the values $p_{s}\left(x_{i}\right)=0$ or $q_{s}\left(x_{i}\right)=0$ for some $x_{i} \in[0,1]$ which make (1) be singular at $x=x_{i}$, whilst $a_{s}(x), b_{s}(x)$ are continuous real-valued functions on $[0,1]$, in which $s=1,2$. Through this paper, we assume that (1) and (2) have a unique solution on $[0,1]$.

A number of theoretical results for the solutions of various types of systems of singular differential equations have been developed over the last couple of decades. The reader is asked to refer to [9-15] in order to know more details about these analyses, including their kinds and history, their modifications and conditions for use, their scientific applications, their importance and characteristics, and their relationship including the differences.
Reproducing kernel theory has important application in numerical analysis, computational mathematics, image processing, machine learning, finance, and probability and statistics [16-19]. Recently, a lot of research work has been devoted to the applications of the reproducing kernel theory representative in the RKHS method, which provides the analytical-numerical solutions for linear and nonlinear problems, for wide classes of stochastic and deterministic problems involving operator equations, differential equations, fuzzy differential equations, integral equations, and integrodifferential equations. The RKHS method was successfully used by many authors to investigate several scientific applications side by side with their theories. The reader is kindly requested to go through [20-38] in order to know more details about RKHS method, including its history, its modification for use, its scientific applications, its kernel functions, and its characteristics.

The rest of the paper is organized as follows. In the next section, several inner product spaces are constructed in order to apply the method. In Section 3, the analytical-numerical solutions and theoretical basis of the method are introduced. In Section 4, an iterative method for the analytical-numerical solutions is described and the $n$-truncation numerical solutions are proved to converge to the analytical solutions. In Section 5, we derive the error estimation and the error bound in order to capture the behavior of the numerical solutions. In order to verify the mathematical simulation of the proposed method, two nonlinear numerical examples are presented in Section 6. Some concluding remarks are presented in Section 7. This paper ends in Appendices, with two parts about the kernel function of the space $W_{2}^{3}[0,1]$.

\section{Building Several Inner Product Spaces}

In functional analysis, the RKHS is a Hilbert space of functions in which pointwise evaluation is a continuous linear functional. Equivalently, they are spaces that can be defined by reproducing kernels. In this section, we utilize the reproducing kernel concept in order to construct the RKHS's $W_{2}^{3}[0,1]$ and $W_{2}^{1}[0,1]$. After that, two reproducing kernels functions $R_{x}(y)$ and $G_{x}(y)$ are building in order to formulate and utilize the analytical-numerical solutions via RKHS technique. Throughout this paper $\mathbb{C}$ is the set of complex numbers, $L^{2}[0,1]=\left\{u \mid \int_{0}^{1} u^{2}(x) d x<\infty\right\}$, and $l^{2}=\left\{A \mid \sum_{i=1}^{\infty}\left(A_{i}\right)^{2}<\infty\right\}$.

Prior to discussing the applicability of the RKHS method on solving singular periodic differential systems and their associated numerical algorithm, it is necessary to present an appropriate brief introduction to preliminary topics from the reproducing kernel theory.

Definition 1 (see [16]). Let $H$ be a Hilbert space of a function $\phi: \Omega \rightarrow \mathscr{F}$ on a set $\Omega$. A function $K: \Omega \times \Omega \rightarrow \mathbb{C}$ is a reproducing kernel of $H$ if the following conditions are satisfied. Firstly, $K(\cdot, x) \in H$ for each $x \in \Omega$. Secondly, $\langle\phi(\cdot), K(\cdot, x)\rangle=\phi(x)$ for each $\phi \in H$ and each $x \in \Omega$.

The second condition in Definition 1 is called "the reproducing property" which means that the value of 
the function $\phi$ at the point $x$ is reproduced by the inner product of $\phi$ with $K(\cdot, x)$. Indeed, a Hilbert space $H$ of functions on a nonempty abstract set $\Omega$ is called a RKHSs, if there exists a reproducing kernel $K$ of $H$.

It is worth mentioning that the reproducing kernel function $K$ of a Hilbert space $H$ is unique, and the existence of $K$ is due to the Riesz representation theorem, where $K$ completely determines the space $H$. Moreover, every sequence of functions $\phi_{1}, \phi_{2}, \ldots, \phi_{n}, \ldots$, which converges strongly to a function $\phi$ in $H$, converges also in the pointwise sense. This convergence is uniform on every subset on $\Omega$ in which $x \rightarrow$ $K(x, x)$ is bounded. In this occasion, these spaces have wide applications including complex analysis, harmonic analysis, quantum mechanics, statistics, and machine learning. For the theoretical background of the reproducing kernel theory and its applications, we refer the reader to [16-38].

Definition 2. The inner product space $W_{2}^{3}[0,1]$ is defined as $W_{2}^{3}[0,1]=\left\{u \mid u, u^{\prime}, u^{\prime \prime}\right.$ are absolutely continuous realvalued functions on $[0,1], u, u^{\prime}, u^{\prime \prime}, u^{\prime \prime \prime} \in L^{2}[0,1]$, and $u(0)=$ $\left.u(1), u^{\prime}(0)=u^{\prime}(1)\right\}$. On the other hand, the inner product and the norm of $W_{2}^{3}[0,1]$ are defined, respectively, by

$$
\begin{aligned}
\langle u(x), v(x)\rangle_{W_{2}^{3}}= & \sum_{i=0}^{2} u^{(i)}(0) v^{(i)}(0) \\
& +\int_{0}^{1} u^{\prime \prime \prime}(x) v^{\prime \prime \prime}(x) d x,
\end{aligned}
$$

and $\|u\|_{W_{2}^{3}}=\sqrt{\langle u(x), u(x)\rangle_{W_{2}^{3}}}$, where $u, v \in W_{2}^{3}[0,1]$.

The Hilbert space $W_{2}^{3}[0,1]$ is called a reproducing kernel if for each fixed $x \in[0,1]$ and any $u \in W_{2}^{3}[0,1]$, there exist $R \in W_{2}^{3}[0,1]$ and $y \in[0,1]$ such that $\langle u(y), R(x, y)\rangle_{W_{2}^{3}}=$ $u(x)$. Henceforth and not to conflict unless stated otherwise, we denote $R(x, y)$ simply by $R_{x}(y)$.

Theorem 3. The Hilbert space $W_{2}^{3}[0,1]$ is a complete reproducing kernel and its reproducing kernel function $R_{x}(y)$ can be written as

$$
R_{x}(y)= \begin{cases}\sum_{i=1}^{6} a_{i}(x) y^{i-1}, & y \leq x \\ \sum_{i=1}^{6} b_{i}(x) y^{i-1}, & y>x\end{cases}
$$

where $a_{i}(x)$ and $b_{i}(x), i=1,2, \ldots, 6$, are unknown coefficients of $R_{x}(y)$.

Proof. The proof and the coefficients of the reproducing kernel function $R_{x}(y)$ are given in Appendices A and $\mathrm{B}$, respectively.

Definition 4 (see [20]). The inner product space $W_{2}^{1}[0,1]$ is defined as $W_{2}^{1}[0,1]=\{u \mid u$ is absolutely continuous realvalued function on $[0,1]$ and $\left.u^{\prime} \in L^{2}[0,1]\right\}$. On the other hand, the inner product and the norm of $W_{2}^{1}[0,1]$ are defined, respectively, by

$$
\langle u(x), v(x)\rangle_{W_{2}^{1}}=u(0) v(0)+\int_{0}^{1} u^{\prime}(x) v^{\prime}(x) d x,
$$

and $\|u\|_{W_{2}^{1}}=\sqrt{\langle u(x), u(x)\rangle_{W_{2}^{1}}}$, where $u, v \in W_{2}^{1}[0,1]$.

Theorem 5 (see [20]). The Hilbert space $W_{2}^{1}[0,1]$ is a complete reproducing kernel and its reproducing kernel function $G_{x}(y)$ can be written as

$$
G_{x}(y)= \begin{cases}a_{1}(x)+a_{2}(x) y, & y \leq x, \\ b_{1}(x)+b_{2}(x) y, & y>x,\end{cases}
$$

where $a_{i}(x)$ and $b_{i}(x), i=1,2$, are unknown coefficients of $G_{x}(y)$ and are given as $a_{1}(x)=1, a_{2}(x)=1, b_{1}(x)=1+x$, and $b_{2}(x)=0$.

The spaces $W_{2}^{1}[0,1]$ and $W_{2}^{3}[0,1]$ are complete Hilbert with some special properties. So, all the properties of the Hilbert space will be held. Further, this space possesses some special and better properties which could make some problems be solved easier. For instance, many problems studied in $L^{2}[0,1]$ space, which is a complete Hilbert space, require large amount of integral computations and such computations may be very difficult in some cases. Thus, the numerical integrals have to be calculated in the cost of losing some accuracy. However, the properties of $W_{2}^{1}[0,1]$ and $W_{2}^{3}[0,1]$ require no more integral computation for some functions, instead of computing some values of a function at some nodes. In fact, this simplification of integral computation not only improves the computational speed, but also improves the computational accuracy.

\section{Formulation of the Analytical-Numerical Solutions}

In this section, formulation of the differential linear operator and implementation method are presented in the space $W_{2}^{3}[0,1]$. Meanwhile, we construct an orthogonal function system based on the Gram-Schmidt orthogonalization process in order to obtain the analytical-numerical solutions. For the remaining sections, the lowercase letter $s$ whenever used means for each $s=1,2$.

To deal with (1) and (2) in more realistic form via the RKHS approach, multiplying both sides of (1) by $p_{s}(x) q_{s}(x)$ and define the differential operator $L$ as

$$
L: W_{2}^{3}[0,1] \longrightarrow W_{2}^{1}[0,1],
$$

such that

$$
\begin{aligned}
L u_{s}(x)= & p_{s}(x) q_{s}(x) u_{s}^{\prime \prime}(x)+q_{s}(x) a_{s}(x) u_{s}^{\prime}(x) \\
& +p_{s}(x) b_{s}(x) u_{s}(x) .
\end{aligned}
$$

As a result, (1) and (2) can be converted into the equivalent form as follows:

$$
L u_{s}(x)=F_{s}\left(x, u_{1}(x), u_{2}(x)\right), \quad 0<x<1,
$$


subject to the periodic boundary conditions

$$
\begin{aligned}
& u_{s}(0)-u_{s}(1)=0, \\
& u_{s}^{\prime}(0)-u_{s}^{\prime}(1)=0,
\end{aligned}
$$

where $F_{s}\left(x, u_{1}(x), u_{2}(x)\right)=p_{s}(x) q_{s}(x) f_{s}\left(x, u_{1}(x), u_{2}(x)\right)$.

Theorem 6. The operator $L: W_{2}^{3}[0,1] \rightarrow W_{2}^{1}[0,1]$ is bounded and linear.

Proof. For boundedness, we need to prove $\left\|L u_{s}\right\|_{W_{2}^{1}} \leq$ $M\left\|u_{s}\right\|_{W_{2}^{3}}$, where $M>0$. From the definition of the inner product and the norm of $W_{2}^{1}[0,1]$, we have $\left\|L u_{s}\right\|_{W_{2}^{1}}^{2}=$ $\left\langle L u_{s}, L u_{s}\right\rangle_{W_{2}^{1}}=\left[\left(L u_{s}\right)(0)\right]^{2}+\int_{0}^{1}\left[\left(L u_{s}\right)^{\prime}(x)\right]^{2} d x$. By the Schwarz inequality and the reproducing properties $u_{s}(x)=$ $\left\langle u_{s}(y), R_{x}(y)\right\rangle_{W_{2}^{3}},\left(L u_{s}\right)(x)=\left\langle u_{s}(y),\left(L R_{x}\right)(y)\right\rangle_{W_{2}^{3}}$, and $\left(L u_{s}\right)^{\prime}(x)=\left\langle u_{s}(y),\left(L R_{x}\right)^{\prime}(y)\right\rangle_{W_{2}^{3}}$ of $R_{x}(y)$, we get

$$
\begin{aligned}
\left|\left(L u_{s}\right)(x)\right| & =\left|\left\langle u_{s}(x),\left(L R_{x}\right)(x)\right\rangle_{W_{2}^{3}}\right| \\
& \leq\left\|L R_{x}\right\|_{W_{2}^{3}}\left\|u_{s}\right\|_{W_{2}^{3}}=M_{1}\left\|u_{s}\right\|_{W_{2}^{3}}, \\
\left|\left(L u_{s}\right)^{\prime}(x)\right| & =\left|\left\langle u_{s}(x),\left(L R_{x}\right)^{\prime}(x)\right\rangle_{W_{2}^{3}}\right| \\
& \leq\left\|\left(L R_{x}\right)^{\prime}\right\|_{W_{2}^{3}}\left\|u_{s}\right\|_{W_{2}^{3}}=M_{2}\left\|u_{s}\right\|_{W_{2}^{3}},
\end{aligned}
$$

where $M_{s}>0$. Thus, $\left\|L u_{s}\right\|_{W_{2}^{1}}^{2}=\left[\left(L u_{s}\right)(0)\right]^{2}+$ $\int_{0}^{1}\left[\left(L u_{s}\right)^{\prime}(x)\right]^{2} d x \leq\left(M_{1}^{2}+M_{2}^{2}\right)\left\|u_{s}\right\|_{W_{2}^{3}}^{2}$ or $\left\|L u_{s}\right\|_{W_{2}^{1}} \leq$ $M\left\|u_{s}\right\|_{W_{2}^{3}}$, where $M=\sqrt{M_{1}^{2}+M_{2}^{2}}$. The linearity part is obvious. The proof is complete.

Next, some theoretic basis of the RKHS method is introduced. Initially, we construct an orthogonal function system of $W_{2}^{3}[0,1]$; to do so, put $\varphi_{i}(x)=G_{x_{i}}(x)$ and $\psi_{i}(x)=L^{*} \varphi_{i}(x)$, where $\left\{x_{i}\right\}_{i=1}^{\infty}$ is dense on $[0,1]$ and $L^{*}$ is the adjoint operator of $L$. In other words, $\left\langle u_{s}(x), \psi_{i}(x)\right\rangle_{W_{2}^{3}}=\left\langle u_{s}(x), L^{*} \varphi_{i}(x)\right\rangle_{W_{2}^{3}}=$ $\left\langle L u_{s}(x), \varphi_{i}(x)\right\rangle_{W_{2}^{1}}=L u_{s}\left(x_{i}\right), i=1,2,3, \ldots$

Algorithm 7. The orthonormal function system $\left\{\bar{\psi}_{i}(x)\right\}_{i=1}^{\infty}$ of the space $W_{2}^{3}[0,1]$ can be derived from the Gram-Schmidt orthogonalization process of $\left\{\psi_{i}(x)\right\}_{i=1}^{\infty}$ as follows:

Step 1 . For $i=1,2,3, \ldots$ and $j=1,2,3, \ldots, i$ do the following:

$$
\text { If } i=j=1 \text {, then set }
$$

$$
\beta_{i j}=\frac{1}{\left\|\psi_{1}\right\|_{W_{2}^{3}}} ;
$$

If $i=j \neq 1$, then set

$$
\beta_{i j}=\frac{1}{\sqrt{\left\|\psi_{i}\right\|_{W_{2}^{3}}^{2}-\sum_{k=1}^{i-1}\left\langle\psi_{i}(x), \bar{\psi}_{k}(x)\right\rangle_{W_{2}^{3}}^{2}}} ;
$$

If $i>j$, then set

$$
\begin{aligned}
\beta_{i j}= & -\frac{1}{\sqrt{\left\|\psi_{i}\right\|_{W_{2}^{3}}^{2}-\sum_{k=1}^{i-1}\left\langle\psi_{i}(x), \bar{\psi}_{k}(x)\right\rangle_{W_{2}^{3}}^{2}}} \\
& \cdot \sum_{k=j}^{i-1}\left\langle\psi_{i}(x), \bar{\psi}_{k}(x)\right\rangle_{W_{2}^{3}} \beta_{k j} ;
\end{aligned}
$$

Output: the orthogonalization coefficients $\beta_{i k}$ of the orthonormal system $\psi_{i}(x)$.

Step 2. For $i=1,2,3, \ldots$ set

$$
\bar{\psi}_{i}(x)=\sum_{k=1}^{i} \beta_{i k} \psi_{k}(x)
$$

Output: the orthonormal function system $\left\{\bar{\psi}_{i}(x)\right\}_{i=1}^{\infty}$.

Step 3. Stop.

It is easy to see that $\psi_{i}(x)=L^{*} \varphi_{i}(x)=\left\langle L^{*} \varphi_{i}(x), R_{x}(y)\right\rangle_{W_{2}^{3}}$ $=\left\langle\varphi_{i}(x), L_{y} R_{x}(y)\right\rangle_{W_{2}^{1}}=\left.L_{y} R_{x}(y)\right|_{y=x_{i}} \in W_{2}^{3}[0,1]$. Thus, $\psi_{i}(x)$ can be written in the form $\psi_{i}(x)=\left.L_{y} R_{x}(y)\right|_{y=x_{i}}$, where $L_{y}$ indicates that the operator $L$ applies to the function of $y$.

Theorem 8. If $\left\{x_{i}\right\}_{i=1}^{\infty}$ is dense on $[0,1]$, then $\left\{\psi_{i}(x)\right\}_{i=1}^{\infty}$ is a complete function system of the space $W_{2}^{3}[0,1]$.

Proof. For each fixed $u_{s} \in W_{2}^{3}[0,1]$, let $\left\langle u_{s}(x), \psi_{i}(x)\right\rangle_{W_{2}^{3}}=$ $0, i=1,2,3, \ldots$. In other words, one has $\left\langle u_{s}(x), \psi_{i}(x)\right\rangle_{W_{2}^{3}}=$ $\left\langle u_{s}(x), L^{*} \varphi_{i}(x)\right\rangle_{W_{2}^{3}}=\left\langle L u_{s}(x), \varphi_{i}(x)\right\rangle_{W_{2}^{1}}=L u_{s}\left(x_{i}\right)=0$. Note that $\left\{x_{i}\right\}_{i=1}^{\infty}$ is dense on $[0,1]$; therefore $L u_{s}(x)=0$. It follows that $u_{s}(x)=0$ from the existence of $L^{-1}$. So, the proof of the theorem is complete.

Lemma 9. If $u_{s} \in W_{2}^{3}[0,1]$, then $\left|u_{s}(x)\right| \leq(7 / 2)\left\|u_{s}\right\|_{W_{2}^{3}}$, $\left|u_{s}^{\prime}(x)\right| \leq 3\left\|u_{s}\right\|_{W_{2}^{3}}$, and $\left|u_{s}^{\prime \prime}(x)\right| \leq 2\left\|u_{s}\right\|_{W_{2}^{3}}$.

Proof. For the first part, note that $u_{s}^{\prime \prime}(x)-u_{s}^{\prime \prime}(0)=$ $\int_{0}^{x} u_{s}^{\prime \prime \prime}(p) d p$, where $u_{s}^{\prime \prime}(x)$ are absolutely continuous on $[0,1]$. If those are integrated again from 0 to $x$, the result is $u_{s}^{\prime}(x)$ itself as $u_{s}^{\prime}(x)-u_{s}^{\prime}(0)-u_{s}^{\prime \prime}(0) x=\int_{0}^{x}\left(\int_{0}^{v} u_{s}^{\prime \prime \prime}(p) d p\right) d v$. Again, integrated from 0 to $x$, yield that $u_{s}(x)-u_{s}(0)-u_{s}^{\prime}(0) x-$ $(1 / 2) u_{s}^{\prime \prime}(0) x^{2}=\int_{0}^{x}\left(\int_{0}^{w}\left(\int_{0}^{v} u_{s}^{\prime \prime \prime}(p) d p\right) d v\right) d w$. So, $\left|u_{s}(x)\right| \leq$ $\left|u_{s}(0)\right|+\left|u_{s}^{\prime}(0)\right||x|+(1 / 2)\left|u_{s}^{\prime \prime}(0)\right||x|^{2}+\int_{0}^{1}\left|u_{s}^{\prime \prime \prime}(p)\right| d p$ or $\left|u_{s}(x)\right| \leq\left|u_{s}(0)\right|+\left|u_{s}^{\prime}(0)\right|+(1 / 2)\left|u_{s}^{\prime \prime}(0)\right|+\int_{0}^{1}\left|u_{s}^{\prime \prime \prime}(p)\right| d p$. By 
using Holder's inequality and (3), one can note the following inequalities:

$$
\begin{aligned}
\left|u_{s}(0)\right| & =\sqrt{u_{s}^{2}(0)} \\
& \leq \sqrt{\sum_{i=0}^{2}\left(u_{s}^{(i)}(0)\right)^{2}+\int_{0}^{1}\left(u_{s}^{\prime \prime \prime}(x)\right)^{2} d x} \\
& =\left\|u_{s}\right\|_{W_{2}^{3}} \\
\left|u_{s}^{\prime}(0)\right| & =\sqrt{\left(u_{s}^{\prime}(0)\right)^{2}} \\
& \leq \sqrt{\sum_{i=0}^{2}\left(u_{s}^{(i)}(0)\right)^{2}+\int_{0}^{1}\left(u_{s}^{\prime \prime \prime}(x)\right)^{2} d x} \\
& =\left\|u_{s}\right\|_{W_{2}^{3}}, \\
\int_{0}^{1}\left|u_{s}^{\prime \prime \prime}(p)\right| d p & \leq \sqrt{\int_{0}^{1}\left(u_{s}^{\prime \prime \prime}(p)\right)^{2} d p \int_{0}^{1}(1)^{2} d p} \\
& =\sqrt{\left(u_{s}^{\prime \prime}(0)\right)^{2}} \\
& \leq \sqrt{\sum_{i=0}^{2}\left(u_{s}^{(i)}(0)\right)^{2}+\int_{0}^{1}\left(u_{s}^{\prime \prime \prime}(x)\right)^{2} d x}
\end{aligned}
$$

Thus, $\left|u_{s}(x)\right| \leq(7 / 2)\left\|u_{s}\right\|_{W_{2}^{3}}$. For the second part, since $u_{s}^{\prime}(x)=u_{s}^{\prime}(0)+u_{s}^{\prime \prime}(0) x+\int_{0}^{x}\left(\int_{0}^{v} u_{s}^{\prime \prime \prime}(p) d p\right) d v$, this means that $\left|u_{s}^{\prime}(x)\right| \leq\left|u_{s}^{\prime}(0)\right|+\left|u_{s}^{\prime \prime}(0)\right|+\int_{0}^{1}\left|u_{s}^{\prime \prime \prime}(p)\right| d p$. Thus, one can find $\left|u_{s}^{\prime}(x)\right| \leq 3\left\|u_{s}\right\|_{W_{2}^{3}}$. In the third part, clearly, $u_{s}^{\prime \prime}(x)-$ $u_{s}^{\prime \prime}(0)=\int_{0}^{x} u_{s}^{\prime \prime \prime}(p) d p$, which yield that $\left|u_{s}^{\prime \prime}(x)\right| \leq\left|u_{s}^{\prime \prime}(0)\right|+$ $\int_{0}^{1}\left|u_{s}^{\prime \prime \prime}(p)\right| d p$. Hence, one can write $\left|u_{s}^{\prime \prime}(x)\right| \leq 2\left\|u_{s}\right\|_{W_{2}^{3}}$.

\section{Iterative Algorithm for the Analytical-Numerical Solutions}

In this section, an iterative algorithm of obtaining the analytical-numerical solutions is represented in the reproducing kernel space $W_{2}^{3}[0,1]$. The numerical solution is obtained by taking finitely many terms in this series representation form. Also, the numerical solutions and their derivatives are proved to converge uniformly to the analytical solution and their derivatives, respectively.

The internal structure of the following theorem is to give the representation form of the analytical solutions. After that, the convergence of the numerical solutions $u_{s, n}(x)$ to the analytical solutions $u_{s}(x)$ will be proved.

Theorem 10. For each $u_{s} \in W_{2}^{3}[0,1]$, the series $\sum_{i=1}^{\infty}\left\langle u_{s}(x), \bar{\psi}_{i}(x)\right\rangle_{W_{2}^{3}} \bar{\psi}_{i}(x)$ are convergent in the sense of the norm of $W_{2}^{3}[0,1]$. On the other hand, if $\left\{x_{i}\right\}_{i=1}^{\infty}$ is dense on $[0,1]$, then the analytical solutions of (9) and (10) could be represented by

$$
u_{s}(x)=\sum_{i=1}^{\infty} \sum_{k=1}^{i} \beta_{i k} F_{s}\left(x_{k}, u_{1}\left(x_{k}\right), u_{2}\left(x_{k}\right)\right) \bar{\psi}_{i}(x) .
$$

Proof. Let $u_{s}(x)$ be solutions of (9) and (10) in $W_{2}^{3}[0,1]$. Since $u_{s} \in W_{2}^{3}[0,1], \sum_{i=1}^{\infty}\left\langle u_{s}(x), \bar{\psi}_{i}(x)\right\rangle_{W_{2}^{3}} \bar{\psi}_{i}(x)$ are the Fourier series expansion about normal orthogonal system $\left\{\bar{\psi}_{i}(x)\right\}_{i=1}^{\infty}$, and $W_{2}^{3}[0,1]$ is the Hilbert space, then the series $\sum_{i=1}^{\infty}\left\langle u_{s}(x), \bar{\psi}_{i}(x)\right\rangle_{W_{2}^{3}} \bar{\psi}_{i}(x)$ are convergent in the sense of $\|\cdot\|_{W_{2}^{3}}$. On the other hand, using (15), yields that

$$
\begin{aligned}
u_{s}(x) & \\
= & \sum_{i=1}^{\infty}\left\langle u_{s}(x), \bar{\psi}_{i}(x)\right\rangle_{W_{2}^{3}} \bar{\psi}_{i}(x) \\
= & \sum_{i=1}^{\infty} \sum_{k=1}^{i} \beta_{i k}\left\langle u_{s}(x), \psi_{k}(x)\right\rangle_{W_{2}^{3}} \bar{\psi}_{i}(x) \\
= & \sum_{i=1}^{\infty} \sum_{k=1}^{i} \beta_{i k}\left\langle u_{s}(x), L_{s}^{*} \varphi_{k}(x)\right\rangle_{W_{2}^{3}} \bar{\psi}_{i}(x) \\
= & \sum_{i=1}^{\infty} \sum_{k=1}^{i} \beta_{i k}\left\langle L_{s} u_{s}(x), \varphi_{k}(x)\right\rangle_{W_{2}^{1}} \bar{\psi}_{i}(x) \\
= & \sum_{i=1}^{\infty} \sum_{k=1}^{i} \beta_{i k}\left\langle F_{s}\left(x, u_{1}(x), u_{2}(x)\right), \varphi_{k}(x)\right\rangle_{W_{2}^{1}} \bar{\psi}_{i}(x) \\
= & \sum_{i=1}^{\infty} \sum_{k=1}^{i} \beta_{i k} F_{s}\left(x_{k}, u_{1}\left(x_{k}\right), u_{2}\left(x_{k}\right)\right) \bar{\psi}_{i}(x) .
\end{aligned}
$$

Therefore, the form of (17) is the analytical solutions of (9) and (10). So, the proof of the theorem is complete.

Let $\left\{\bar{\psi}_{i}(x)\right\}_{i=1}^{\infty}$ be the normal orthogonal system derived from the Gram-Schmidt orthogonalization process of $\left\{\psi_{i}(x)\right\}_{i=1}^{\infty}$. Then according to (17), the analytical solution of (9) and (10) can be denoted by

$$
u_{s}(x)=\sum_{i=1}^{\infty} B_{i}^{\{s\}} \bar{\psi}_{i}(x)
$$

where $B_{i}^{\{s\}}=\sum_{k=1}^{i} \beta_{i k} F_{s}\left(x_{k}, u_{1, k-1}\left(x_{k}\right), u_{2, k-1}\left(x_{k}\right)\right)$. In fact, $B_{i}^{\{s\}}, i=1,2,3, \ldots$, are unknown; we will approximate $B_{i}^{\{s\}}$ using known $A_{i}^{\{s\}}$. For numerical computations, define initial functions $u_{s, 0}\left(x_{1}\right)=0$, put $u_{s, 0}\left(x_{1}\right)=u_{s}\left(x_{1}\right)$, and set the $n$ term numerical approximations to $u_{s}(x)$ by

$$
u_{s, n}(x)=\sum_{i=1}^{n} A_{i}^{\{s\}} \bar{\psi}_{i}(x),
$$


where the coefficients $A_{i}^{\{s\}}$ of the normal orthogonal system $\bar{\psi}_{i}(x)$ are given as

$$
A_{i}^{\{s\}}=\sum_{k=1}^{i} \beta_{i k} F_{s}\left(x_{k}, u_{1, k-1}\left(x_{k}\right), u_{2, k-1}\left(x_{k}\right)\right) .
$$

According to Lemma 9, it is clear that, for any $x \in[0,1]$, the analytical-numerical solutions of (9) and (10) satisfy

$$
\left|u_{s, n}^{(i)}(x)-u_{s}^{(i)}(x)\right| \leq M_{i}\left\|u_{s, n}-u_{s}\right\|_{W_{2}^{3}}, \quad i=0,1,2,
$$

where $M_{0}=7 / 2, M_{1}=3$, and $M_{2}=2$. As a result, if $\left\|u_{s, n}-u_{s}\right\|_{W_{2}^{3}} \rightarrow 0$ as $n \rightarrow \infty$, then the numerical solutions $u_{s, n}(x)$ and $u_{s, n}^{(i)}(x)$ are converged uniformly to the analytical solutions $u_{s}(x)$ and $u_{s}^{(i)}(x), i=0,1,2$, respectively.

Lemma 11. If $\left\|u_{s, n-1}-u_{s}\right\|_{W_{2}^{3}} \rightarrow 0, x_{n} \rightarrow y$ as $n \rightarrow \infty$, and $F_{s}\left(x, v_{1}, v_{2}\right)$ is continuous in $[0,1]$ with respect to $x, v_{s}$ for $x \in[0,1]$ and $v_{s} \in(-\infty, \infty)$, then $F_{s}\left(x_{n}, u_{1, n-1}\left(x_{n}\right), u_{2, n-1}\left(x_{n}\right)\right) \rightarrow F_{s}\left(y, u_{1}(y), u_{2}(y)\right)$ as $n \rightarrow$ $\infty$.

Proof. Firstly, we will prove that $u_{s, n-1}\left(x_{n}\right) \rightarrow u_{s}(y)$ in the sense of $\|\cdot\|_{W_{2}^{3}}$. Since

$$
\begin{aligned}
& \left|u_{s, n-1}\left(x_{n}\right)-u_{s}(y)\right| \\
& \quad=\left|u_{s, n-1}\left(x_{n}\right)-u_{s, n-1}(y)+u_{s, n-1}(y)-u_{s}(y)\right| \\
& \quad \leq\left|u_{s, n-1}\left(x_{n}\right)-u_{s, n-1}(y)\right|+\left|u_{s, n-1}(y)-u_{s}(y)\right| .
\end{aligned}
$$

By reproducing property of $R_{x}(y)$, we have $u_{s, n-1}\left(x_{n}\right)=$ $\left\langle u_{s, n-1}(x), R_{x_{n}}(x)\right\rangle$ and $u_{s, n-1}(y)=\left\langle u_{s, n-1}(x), R_{y}(x)\right\rangle$. Thus, $\left|u_{s, n-1}\left(x_{n}\right)-u_{s, n-1}(y)\right|=\left|\left\langle u_{s, n-1}(x), R_{x_{n}}(x)-R_{y}(x)\right\rangle_{W_{2}^{3}}\right| \leq$ $\left\|u_{s, n-1}\right\|_{W_{2}^{3}}\left\|R_{x_{n}}-R_{y}\right\|_{W_{2}^{3}}$. From the symmetry of $R$, it follows that $\left\|R_{x_{n}}-R_{y}\right\|_{W_{2}^{3}} \rightarrow 0$ as $n \rightarrow \infty$. Hence, $\mid u_{s, n-1}\left(x_{n}\right)-$ $u_{s, n-1}(y) \mid \rightarrow 0$ as soon as $x_{n} \rightarrow y$. On the other hand, by Lemma 9, for any $y \in[0,1]$ it holds that $\left|u_{s, n-1}(y)-u_{s}(y)\right| \leq$ $(7 / 2)\left\|u_{s, n-1}-u_{s}\right\|_{W_{2}^{3}} \rightarrow 0$ as $n \rightarrow \infty$. Therefore, $u_{s, n-1}\left(x_{n}\right) \rightarrow$ $u_{s}(y)$ in the sense of $\|\cdot\|_{W_{2}^{3}}$ as $x_{n} \rightarrow y$ and $n \rightarrow \infty$. Thus, by means of the continuation of $F_{s}$ it is obtained that $F_{s}\left(x_{n}, u_{1, n-1}\left(x_{n}\right), u_{2, n-1}\left(x_{n}\right)\right) \rightarrow F_{s}\left(y, u_{1}(y), u_{2}(y)\right)$ as $n \rightarrow$ $\infty$.

Lemma 12. One has $L u_{s, n}\left(x_{j}\right)=L u_{s}\left(x_{j}\right)=$ $F_{s}\left(x_{j}, u_{1, j-1}\left(x_{j}\right), u_{2, j-1}\left(x_{j}\right)\right)$ as $j \leq n$.

Proof. The proof of $\operatorname{Lu}_{s, n}\left(x_{j}\right)=F_{s}\left(x_{j}, u_{1, j-1}\left(x_{j}\right), u_{2, j-1}\left(x_{j}\right)\right)$ is obtained by using the mathematical induction as follows: if $j \leq n$, then $L u_{s, n}\left(x_{j}\right)=\sum_{i=1}^{n} A_{i}^{\{s\}} L \bar{\psi}_{i}\left(x_{j}\right)$ $=\sum_{i=1}^{n} A_{i}^{\{s\}}\left\langle L \bar{\psi}_{i}(x), \varphi_{j}(x)\right\rangle_{W_{2}^{1}}=\sum_{i=1}^{n} A_{i}^{\{s\}}\left\langle\bar{\psi}_{i}(x), L_{j}^{*} \varphi(x)\right\rangle_{W_{2}^{3}}$ $=\sum_{i=1}^{n} A_{i}^{\{s\}}\left\langle\bar{\psi}_{i}(x), \psi_{j}(x)\right\rangle_{W_{2}^{3}}$. Using the orthogonality of $\left\{\bar{\psi}_{i}(x)\right\}_{i=1}^{\infty}$, yields that

$$
\begin{aligned}
\sum_{l=1}^{j} \beta_{j l} L u_{s, n}\left(x_{l}\right) & =\sum_{i=1}^{n} A_{i}^{\{s\}}\left\langle\bar{\psi}_{i}(x), \sum_{l=1}^{j} \beta_{j l} \psi_{l}(x)\right\rangle_{W_{2}^{3}} \\
& =\sum_{i=1}^{n} A_{i}^{\{s\}}\left\langle\bar{\psi}_{i}(x), \bar{\psi}_{j}(x)\right\rangle_{W_{2}^{3}}=A_{j}^{\{s\}} \\
& =\sum_{l=1}^{j} \beta_{j l}^{\{s\}} F_{s}\left(x_{l}, u_{1, l-1}\left(x_{l}\right), u_{2, l-1}\left(x_{l}\right)\right) .
\end{aligned}
$$

Now, if $j=1$, then $L u_{s, n}\left(x_{1}\right)=F_{s}\left(x_{1}, u_{1,0}\left(x_{1}\right), u_{2,0}\left(x_{1}\right)\right)$. Again, if $j=2$, then $\beta_{21} L u_{s, n}\left(x_{1}\right)+\beta_{22} L u_{s, n}\left(x_{2}\right)$ $=\beta_{21} F_{s}\left(x_{1}, u_{1,0}\left(x_{1}\right), u_{2,0}\left(x_{1}\right)\right)+\beta_{22} F_{s}\left(x_{2}, u_{1,1}\left(x_{2}\right), u_{2,1}\left(x_{2}\right)\right)$. Thus, $L u_{s, n}\left(x_{2}\right)=F_{s}\left(x_{2}, u_{1,1}\left(x_{2}\right), u_{2,1}\left(x_{2}\right)\right)$. It is easy to see that $L u_{s, n}\left(x_{j}\right)=F_{s}\left(x_{j}, u_{1, j-1}\left(x_{j}\right), u_{2, j-1}\left(x_{j}\right)\right)$. But on the other aspect as well, from (22) $u_{s, n}(x)$ converge uniformly to $u_{s}(x)$. It follows that, on taking limits in (20), $u_{s}(x)=\sum_{i=1}^{\infty} A_{i}^{\{s\}} \bar{\psi}_{i}(x)$. Therefore, $u_{s, n}(x)=P_{n} u_{s}(x)$, where $P_{n}$ is an orthogonal projector from $W_{2}^{3}[0,1]$ to $\operatorname{Span}\left\{\psi_{1}, \psi_{2}, \ldots, \psi_{n}\right\}$. Thus,

$$
\begin{aligned}
L u_{s, n}\left(x_{j}\right) & =\left\langle L u_{s, n}(x), \varphi_{j}(x)\right\rangle_{W_{2}^{1}} \\
& =\left\langle u_{s, n}(x), L_{j}^{*} \varphi(x)\right\rangle_{W_{2}^{3}} \\
& =\left\langle P_{n} u_{s}(x), \psi_{j}(x)\right\rangle_{W_{2}^{3}} \\
& =\left\langle u_{s}(x), P_{n} \psi_{j}(x)\right\rangle_{W_{2}^{3}} \\
& =\left\langle u_{s}(x), \psi_{j}(x)\right\rangle_{W_{2}^{3}} \\
& =\left\langle L u_{s}(x), \varphi_{j}(x)\right\rangle_{W_{2}^{1}}=L u_{s}\left(x_{j}\right) .
\end{aligned}
$$

Theorem 13. If $\left\|u_{s, n}\right\|_{W_{2}^{3}}$ are bounded and $\left\{x_{i}\right\}_{i=1}^{\infty}$ is dense on $[0,1]$, then the $n$-term numerical solutions $u_{s, n}(x)$ in the iterative formula (20) converges to the analytical solutions $u_{s}(x)$ of (9) and (10) in the space $W_{2}^{3}[0,1]$ and $u_{s}(x)=$ $\sum_{i=1}^{\infty} A_{i}^{\{s\}} \bar{\psi}_{i}(x)$, where $A_{i}^{\{s\}}$ are given by (21).

Proof. The proof consists of the following steps: firstly, we will prove that the sequences $\left\{u_{s, n}\right\}_{n=1}^{\infty}$ in (20) are increasing in the sense of $\|\cdot\|_{W_{2}^{3}}$. By Theorem $8,\left\{\bar{\psi}_{i}\right\}_{i=1}^{\infty}$ is the complete orthonormal system in $W_{2}^{3}[0,1]$. Hence, we have

$$
\begin{aligned}
\left\|u_{s, n}\right\|_{W_{2}^{3}}^{2} & =\left\langle u_{s, n}(x), u_{s, n}(x)\right\rangle_{W_{2}^{3}} \\
& =\left\langle\sum_{i=1}^{n} A_{i}^{\{s\}} \bar{\psi}_{i}(x), \sum_{i=1}^{n} A_{i}^{\{s\}} \bar{\psi}_{i}(x)\right\rangle_{W_{2}^{3}} \\
& =\sum_{i=1}^{n}\left(A_{i}^{\{s\}}\right)^{2} .
\end{aligned}
$$

Therefore, $\left\|u_{s, n}\right\|_{W_{2}^{3}}$ are increasing. 
Secondly, we will prove the convergence of $u_{s, n}(x)$. From (20), we have $u_{s, n+1}(x)=u_{s, n}(x)+A_{n+1}^{\{s\}} \bar{\psi}_{n+1}(x)$. From the orthogonality of $\left\{\bar{\psi}_{i}(x)\right\}_{i=1}^{\infty}$, it follows that $\left\|u_{s, n+1}\right\|_{W_{2}^{3}}^{2}=$ $\left\|u_{s, n}\right\|_{W_{2}^{3}}^{2}+\left(A_{n+1}^{\{s\}}\right)^{2}=\left\|u_{s, n-1}\right\|_{W_{2}^{3}}^{2}+\left(A_{n}^{\{s\}}\right)^{2}+\left(A_{n+1}^{\{s\}}\right)^{2}=\cdots=$ $\left\|u_{s, 0}\right\|_{W_{2}^{3}}^{2}+\sum_{i=1}^{n+1}\left(A_{i}^{\{s\}}\right)^{2}$. Since the sequences $\left\|u_{s, n}\right\|_{W_{2}^{3}}^{2}$ are increasing. Due to the condition that $\left\|u_{s, n}\right\|_{W_{2}^{3}}$ are bounded, $\left\|u_{s, n}\right\|_{W_{2}^{3}}$ are convergent as $n \rightarrow \infty$. Then, there exists constants $c^{\{s\}}$ such that $\sum_{i=1}^{\infty}\left(A_{i}^{\{s\}}\right)^{2}=c^{\{s\}}$. It implies that $A_{i}^{\{s\}}=$ $\sum_{k=1}^{i} \beta_{i k} F_{s}\left(x_{k}, u_{1, k-1}\left(x_{k}\right), u_{2, k-1}\left(x_{k}\right)\right) \in l^{2}, i=1,2,3, \ldots$ On the other hand, since $\left(u_{s, m}-u_{s, m-1}\right) \perp\left(u_{s, m-1}-u_{s, m-2}\right) \perp$ $\cdots \perp\left(u_{s, n+1}-u_{s, n}\right)$ it follows for $m>n$ that

$$
\begin{aligned}
& \left\|u_{s, m}-u_{s, n}\right\|_{W_{2}^{3}}^{2} \\
& =\left\|u_{s, m}-u_{s, m-1}+u_{s, m-1}-\cdots+u_{s, n+1}-u_{s, n}\right\|_{W_{2}^{3}}^{2} \\
& =\left\|u_{s, m}-u_{s, m-1}\right\|_{W_{2}^{3}}^{2}+\cdots+\left\|u_{s, n+1}-u_{s, n}\right\|_{W_{2}^{3}}^{2} \cdot
\end{aligned}
$$

Furthermore, $\left\|u_{s, m}-u_{s, m-1}\right\|_{W_{2}^{3}}^{2}=\left(A_{m}^{\{s\}}\right)^{2}$. Consequently, as $n, m \rightarrow \infty$, we have $\left\|u_{s, m}-u_{s, n}\right\|_{W_{2}^{3}}^{2}=\sum_{i=n+1}^{m}\left(A_{i}^{\{s\}}\right)^{2} \rightarrow$ 0 . Considering the completeness of $W_{2}^{3}[0,1]$, there exists $u_{s}(x) \in W_{2}^{3}[0,1]$ such that $u_{s, n}(x) \rightarrow u_{s}(x)$ as $n \rightarrow \infty$ in the sense of $\|\cdot\|_{W_{2}^{3}}$.

Thirdly, we will prove that $u_{s}(x)$ are the analytical solutions of (9) and (10). Since $\left\{x_{i}\right\}_{i=1}^{\infty}$ is dense on $[0,1]$, for any $x \in[0,1]$, there exists a subsequence $\left\{x_{n_{j}}\right\}_{j=1}^{\infty}$, such that $x_{n_{j}} \rightarrow x$ as $j \rightarrow \infty$. From Lemma 12, It is clear that $L u_{s}\left(x_{n_{j}}\right)=F_{s}\left(x_{n_{j}}, u_{1, n_{j}-1}\left(x_{k}\right), u_{2, n_{j}-1}\left(x_{k}\right)\right)$. Hence, let $j \rightarrow \infty$; by Lemma 11 and the continuity of $F_{s}$, we have $L u_{s}(x)=F_{s}\left(x, u_{1}(x), u_{2}(x)\right)$. That is, $u_{s}(x)$ satisfies (9). Also, since $\bar{\psi}_{i}(x) \in W_{2}^{3}[0,1]$, clearly, $u_{s}(x)$ satisfies the periodic boundary conditions of (10). In other words, $u_{s}(x)$ are the analytical solutions of (9) and (10), where $u_{s}(x)=\sum_{i=1}^{\infty} A_{i}^{\{s\}} \bar{\psi}_{i}(x)$ and $A_{i}^{\{s\}}$ are given by (21). The proof is complete.

\section{Error Estimation and Error Bound}

When solving practical problems, it is necessary to take into account all the errors of the measurements. Moreover, in accordance with the technical progress and the degree of complexity of the problem, it becomes necessary to improve the technique of measurement of quantities. Considerable errors of measurement become inadmissible in solving complicated mathematical, physical, and engineering problems. The reliability of the numerical result will depend on an error estimate or bound, therefore the analysis of error and the sources of error in numerical methods is also a critically important part of the study of numerical technique. In this section, we derive error bounds for the present method and problem in order to capture behavior of the solutions.

In the next theorem, we show that the error of the approximate solutions is decreasing, while the next lemma is presented in order to prove the recent theorem.
Theorem 14. Let $\varepsilon_{s, n}=\left\|u_{s}-u_{s, n}\right\|_{W_{2}^{3}}$, where $u_{s}(x)$ and $u_{s, n}(x)$ are given by (19) and (20), respectively. Then, the sequences $\left\{\varepsilon_{s, n}\right\}$ are decreasing in the sense of the norm of $W_{2}^{3}[0,1]$ and $\varepsilon_{s, n} \rightarrow 0$ as $n \rightarrow \infty$.

Proof. Based on the previous results, it is obvious that

$$
\begin{aligned}
\varepsilon_{s, n}^{2} & =\left\|\sum_{i=n+1}^{\infty}\left\langle u_{s}(x), \bar{\psi}_{i}(x)\right\rangle_{W_{2}^{3}} \bar{\psi}_{i}(x)\right\|_{W_{2}^{3}}^{2} \\
& =\sum_{i=n+1}^{\infty}\left(\left\langle u_{s}(x), \bar{\psi}_{i}(x)\right\rangle_{W_{2}^{3}}\right)^{2}, \\
\varepsilon_{s, n-1}^{2} & =\left\|\sum_{i=n}^{\infty}\left\langle u_{s}(x), \bar{\psi}_{i}(x)\right\rangle_{W_{2}^{3}} \bar{\psi}_{i}(x)\right\|_{W_{2}^{3}}^{2} \\
& =\sum_{i=n}^{\infty}\left(\left\langle u_{s}(x), \bar{\psi}_{i}(x)\right\rangle_{W_{2}^{3}}\right)^{2} .
\end{aligned}
$$

Clearly, $\varepsilon_{s, n-1} \geq \varepsilon_{s, n}$, and consequently $\left\{\varepsilon_{s, n}\right\}$ are decreasing in the sense of $\|\cdot\|_{W_{2}^{3}}$. On the other aspect as well, by Theorem 10, $\sum_{i=1}^{\infty}\left\langle u_{s}(x), \bar{\psi}_{i}(x)\right\rangle_{W_{2}^{3}} \bar{\psi}_{i}(x)$ are convergent, so, $\varepsilon_{s, n}^{2}=\sum_{i=n+1}^{\infty}\left(\left\langle u_{s}(x), \bar{\psi}_{i}(x)\right\rangle_{W_{2}^{3}}\right)^{2} \rightarrow 0$ or $\varepsilon_{s, n} \rightarrow 0$ as $n \rightarrow \infty$. This completes the proof.

Lemma 15. Let $u_{s}(x)$ be the analytical solutions of (9) and (10), and $u_{s, n}(x)$ are the numerical solutions of $u_{s}(x)$. Suppose that $T=\left\{x_{k+1}=k / 2^{i}: k=0,1, \ldots, 2^{i}\right\}$ is the subset of $[0,1]$, where $T$ is the dense subset in $[0,1]$ as $i \rightarrow \infty$. Then, $L u_{s}\left(x_{k}\right)=L u_{s, n}\left(x_{k}\right)$, for $n=2^{i}+1$ and $x_{k} \in T$.

Proof. Set the projective operator $P_{n}: W_{2}^{3}[0,1] \rightarrow$ $\left\{\sum_{m=1}^{n} c_{m}^{\{s\}} \psi_{m}(x), c_{m}^{\{s\}} \in \mathbb{R}\right\}$. Then, we have $L u_{s, n}\left(x_{k}\right)=\left\langle u_{s, n}(\xi), L_{x_{k}} R_{x_{k}}(\xi)\right\rangle_{W_{2}^{3}}=\left\langle u_{s, n}(\xi), \psi_{k}(\xi)\right\rangle_{W_{2}^{3}}=$ $\left\langle P_{n} u_{s}(\xi), \psi_{k}(\xi)\right\rangle_{W_{2}^{3}}=\left\langle u_{s}(\xi), P_{n} \psi_{k}(\xi)\right\rangle_{W_{2}^{3}}=\left\langle u_{s}(\xi), \psi_{k}(\xi)\right\rangle_{W_{2}^{3}}$ $=\left\langle u_{s}(\xi), L_{x_{k}} R_{x_{k}}(\xi)\right\rangle_{W_{2}^{3}}=L_{x_{k}}\left\langle u_{s}(\xi), R_{x_{k}}(\xi)\right\rangle_{W_{2}^{3}}=$ $L_{x_{k}} u_{s}\left(x_{k}\right)=L u_{s}\left(x_{k}\right)$.

Theorem 16. Let $u_{s}(x)$ be the analytical solutions of (9) and (10), and $u_{s, n}(x)$ are the numerical solutions of $u_{s}(x)$. Suppose that $T=\left\{x_{k+1}=k / 2^{i}: k=0,1, \ldots, 2^{i}\right\}$ is the subset of $[0,1]$, where $T$ is the dense subset in $[0,1]$ as $i \rightarrow \infty$. Then, $\mid u_{s}(x)-$ $u_{s, n}(x) \mid<M^{\{s\}} / n$, where $M^{\{s\}}$ are the product of the maximum of determinate function $\left\|(\partial / \partial \eta) R_{\eta}\right\|_{W_{2}^{3}}$ and the sup of convergent basis $\left\|\sum_{i=n+1}^{\infty} \sum_{k=1}^{i} \beta_{i k} F_{s}\left(\xi_{k}, u_{1}\left(\xi_{k}\right), u_{2}\left(\xi_{k}\right)\right) \bar{\psi}_{i}(\xi)\right\|_{W_{2}^{3}}$ about the variable in $[0,1]$.

Proof. Since $\left|u_{s}(x)-u_{s, n}(x)\right|=\left|L^{-1}\left(L u_{s}(x)-L u_{s, n}(x)\right)\right|$ and for every given $x \in[0,1]$, there is always $x_{0} \in T$ satisfying $x_{0}<x$ and $x-x_{0}=1 / n$. On the other hand, Lemma 15 and $x_{0} \in T$ imply that $L u_{s}\left(x_{0}\right)=L u_{s, n}\left(x_{0}\right)$. So, we obtain

$$
\begin{aligned}
& \left|L u_{s}(x)-L u_{s, n}(x)\right| \\
& \quad=\left|\left(L u_{s}(x)-L u_{s}\left(x_{0}\right)\right)-\left(L u_{s, n}(x)-L u_{s, n}\left(x_{0}\right)\right)\right| .
\end{aligned}
$$


By applying the reproducing kernel properties $u_{s}(x)=$ $\left\langle u_{s}(\xi), R_{x}(\xi)\right\rangle_{W_{2}^{3}}$ and $L u_{s}(x)=\left\langle u_{s}(\xi), L R_{x}(\xi)\right\rangle_{W_{2}^{3}}$ to (30), we conclude

$$
\begin{aligned}
& L u_{s}(x)-L u_{s, n}(x) \\
&=\left(L u_{s}(x)-L u_{s}\left(x_{0}\right)\right)-\left(L u_{s, n}(x)-L u_{s, n}\left(x_{0}\right)\right) \\
&=\left\langle u_{s}(\xi), L R_{x}(\xi)-L R_{x_{0}}(\xi)\right\rangle_{W_{2}^{3}} \\
&-\left\langle u_{s, n}(\xi), L R_{x}(\xi)-L R_{x_{0}}(\xi)\right\rangle_{W_{2}^{3}} \\
&=\left\langle u_{s}(\xi)-u_{s, n}(\xi), L R_{x}(\xi)-L R_{x_{0}}(\xi)\right\rangle_{W_{2}^{3}},
\end{aligned}
$$

whilst on the other aspect as well,

$$
\begin{aligned}
& \left|u_{s}(x)-u_{s, n}(x)\right| \\
& \quad=\left|L^{-1}\left(L u_{s}(x)-L u_{s, n}(x)\right)\right| \\
& \quad \leq\left|\left\langle u_{s}(\xi)-u_{s, n}(\xi), L^{-1} L R_{x}(\xi)-L^{-1} L R_{x_{0}}(\xi)\right\rangle_{W_{2}^{3}}\right| \\
& \quad=\left|\left\langle u_{s}(\xi)-u_{s, n}(\xi), R_{x}(\xi)-R_{x_{0}}(\xi)\right\rangle_{W_{2}^{3}}\right| \\
& \quad \leq\left\|u_{s}-u_{s, n}\right\|_{W_{2}^{3}}\left\|R_{x}-R_{x_{0}}\right\|_{W_{2}^{3}} .
\end{aligned}
$$

Here, we take the norm of $\left\|R_{x}-R_{x_{0}}\right\|_{W_{2}^{3}}$ for the variable $\xi$ and the function $R_{x}(\xi)$ is derived on $x$ in $[0,1]$. So, we have $R_{x}(\xi)-R_{x_{0}}(\xi)=(\partial / \partial \eta) R_{\eta}(\xi)\left(x-x_{0}\right)$. Hence, we obtain

$$
\begin{aligned}
& \left|u_{s}(x)-u_{s, n}(x)\right| \leq\left\|u_{s}-u_{s, n}\right\|_{W_{2}^{3}}\left\|\left(x-x_{0}\right) \frac{\partial}{\partial \eta} R_{\eta}\right\|_{W_{2}^{3}} \\
& =\left\|u_{s}-u_{s, n}\right\|_{W_{2}^{3}}\left\|\frac{\partial}{\partial \eta} R_{\eta}\right\|_{W_{2}^{3}}\left(x-x_{0}\right) \\
& =\frac{1}{n}\left\|\sum_{i=n+1}^{\infty} \sum_{k=1}^{i} \beta_{i k} F_{s}\left(\xi_{k}, u_{1}\left(\xi_{k}\right), u_{2}\left(\xi_{k}\right)\right) \bar{\psi}_{i}(\xi)\right\|_{W_{2}^{3}} \\
& \cdot\left\|\frac{\partial}{\partial \eta} R_{\eta}\right\|_{W_{2}^{3}}=\frac{M^{\{s\}}}{n} .
\end{aligned}
$$

So, the proof of the theorem is complete.

\section{Numerical Algorithm and Numerical Outcomes}

In this final section, we consider two nonlinear examples in order to illustrate the performance of the RKHS algorithm in finding the numerical solutions for systems of singular periodic BVPs and justify the accuracy and applicability of the method. These examples have been solved by the presented algorithm while the results obtained are compared with the analytical solutions of each example by computing the absolute and the relative errors and are found to be in good agreement with each other. In the process of computation, all the symbolic and numerical computations performed by using Maple 13 software package.

An algorithm is a precisely defined sequence of steps for performing a specified task. The aim of the next algorithm is to implement a procedure to solve periodic singular differential systems in numeric form in terms of their grid nodes based on the use of RKHS method.

Algorithm 17. To find the numerical solutions $u_{s, n}(x)$ of $u_{s}(x)$ for (9) and (10), we do the following steps:

Input. The endpoints of $[0,1]$, the integers $n$, and the kernel functions $R_{x}(y)$ and $G_{x}(y)$.

Output. Numerical solutions $u_{s, n}(x)$ of $u_{s}(x)$.

Step 1. Fixed $x$ in $[0,1]$ and set $y \in[0,1]$;

$$
\text { If } \begin{aligned}
y \leq x, \text { set } R_{x}(y) & =\sum_{i=1}^{6} a_{i}(x) y^{i-1} ; \\
& \text { else set } R_{x}(y)=\sum_{i=1}^{6} b_{i}(x) y^{i-1} ;
\end{aligned}
$$

For $i=1,2, \ldots, n$ do the following:

$$
\text { Set } x_{i}=(i-1) /(n-1) \text {; }
$$$$
\text { Set } \psi_{i}(x)=L_{y}\left[R_{x}(y)\right]_{y=x_{i}} ;
$$

Output: the orthogonal function system $\psi_{i}(x)$.

Step 2. For $l=2,3, \ldots, n$ and $k=1,2, \ldots, l$, do Algorithm 7 for $l$ and $k$;

Output: the orthogonalization coefficients $\beta_{l k}$.

Step 3. For $l=2,3, \ldots, n-1$ and $k=1,2, \ldots, l-1$, do the following:

$$
\text { Set } \bar{\psi}_{i}(x)=\sum_{l=1}^{i} \beta_{l k} \psi_{l}(x) ;
$$

Output: the orthonormal function system $\bar{\psi}_{i}(x)$.

Step 4. Set $u_{s, 0}\left(x_{1}\right)=u_{s}\left(x_{1}\right)=0$;

Set $A_{i}^{\{s\}}=\sum_{l=1}^{i} \beta_{l k} F_{s}\left(x_{l}, u_{1, l-1}\left(x_{l}\right), u_{2, l-1}\left(x_{l}\right)\right)$;

Set $u_{s, i}(x)=\sum_{i=1}^{n} A_{i}^{\{s\}} \bar{\psi}_{i}(x)$;

Output: the numerical solutions $u_{s, n}(x)$ of $u_{s}(x)$.

Step 5. Stop.

Using RKHS method, take $x_{i}=(i-1) /(n-1), i=$ $1,2, \ldots, n$, with the reproducing kernel functions $R_{x}(y)$ and $G_{x}(y)$ on $[0,1]$ in which Algorithms 7 and 17 are used throughout the computations; some graphical results and tabulated data are presented and discussed quantitatively at some selected grid points on $[0,1]$ to illustrate the numerical solutions for the following periodic singular differential systems.

Example 18. Consider the nonlinear differential system:

$$
\begin{aligned}
& u_{1}^{\prime \prime}(x)+\frac{2}{x(x-1)} u_{1}^{\prime}(x)+\frac{u_{2}(x)}{1+\left(u_{2}(x)\right)^{2}}=f_{1}(x) \\
& u_{2}^{\prime \prime}(x)+\frac{1}{\sin (x)} u_{2}^{\prime}(x)+\frac{1}{x^{3}(x-1)^{3}} e^{x} u_{1}(x) \\
& \quad+x\left(u_{2}(x)\right)^{2}=f_{2}(x)
\end{aligned}
$$




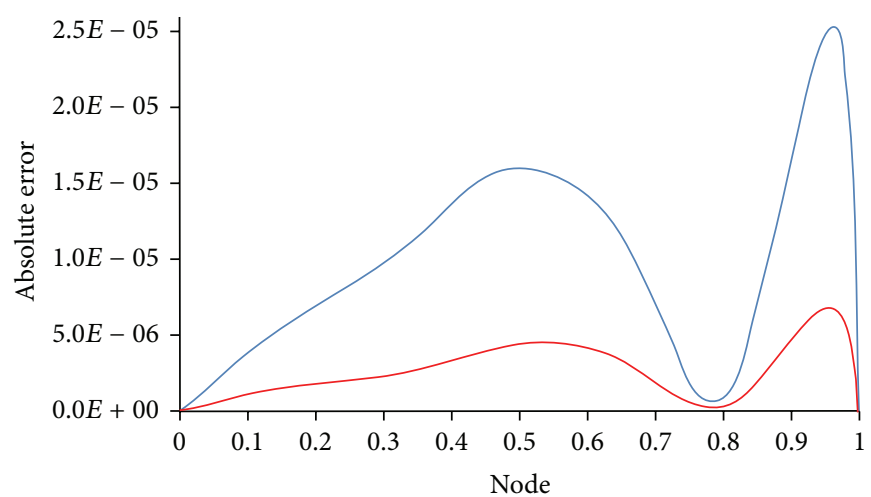

FIGURE 1: The numerical values of the absolute error function for the first derivative of Example 18: blue: the first dependent variable and red: the second dependent variable.

subject to the periodic boundary conditions:

$$
\begin{aligned}
& u_{1}(0)=u_{1}(1), \\
& u_{2}(0)=u_{2}(1), \\
& u_{1}^{\prime}(0)=u_{1}^{\prime}(1), \\
& u_{2}^{\prime}(0)=u_{2}^{\prime}(1),
\end{aligned}
$$

where $x \in(0,1)$ in which $f_{1}(x)$ and $f_{2}(x)$ are chosen such that the analytical solutions are $u_{1}(x)=x^{4}-2 x^{3}+x^{2}$ and $u_{2}(x)=\cos \left(x^{2}-x\right)$.

Example 19. Consider the nonlinear differential system:

$$
\begin{aligned}
& u_{1}^{\prime \prime}(x)+\frac{2}{\ln (x)} u_{1}^{\prime}(x)+\sinh \left(u_{1}(x)-x^{2}(2 x-3)\right) \\
& -u_{2}^{2}(x)=f_{1}(x) \\
& u_{2}^{\prime \prime}(x)+\frac{x^{3}}{\sinh (x)(1-x)} u_{2}^{\prime}(x) \\
& \quad+\exp \left(-u_{2}(x)\right)+u_{1}^{3}(x)=f_{2}(x)
\end{aligned}
$$

subject to the periodic boundary conditions:

$$
\begin{aligned}
& u_{1}(0)=u_{1}(1), \\
& u_{2}(0)=u_{2}(1), \\
& u_{1}^{\prime}(0)=u_{1}^{\prime}(1), \\
& u_{2}^{\prime}(0)=u_{2}^{\prime}(1),
\end{aligned}
$$

where $x \in(0,1)$ in which $f_{1}(x)$ and $f_{2}(x)$ are chosen such that the analytical solutions are $u_{1}(x)=2 x^{3}-3 x^{2}+x$ and $u_{2}(x)=\ln \left(x^{4}-2 x^{3}+x^{2}+1\right)$.

Results from numerical analysis are an approximation, in general, which can be made as accurate as desired. Because a computer has a finite word length, only a fixed number of digits are stored and used during computations. Next, the agreement between the analytical-numerical solutions is investigated for Examples 18 and 19 at various $x$ in $[0,1]$ by computing the absolute errors and the relative errors of numerically approximating their analytical solutions for the corresponding equivalent system as shown in Tables 1, 2, 3, and 4 , respectively.

Anyhow, it is clear from the tables that, the numerical solutions are in close agreement with the analytical solutions, while the accuracy is advanced by using only few tens of the RKHS iterations. Indeed, we can conclude that higher accuracy can be achieved by computing further RKHS iterations. As a computational conclusion, it is to be noted from the tables that the two dependent solutions are relatively of the same order of errors on average for the absolute and the relative error, respectively, for the two examples.

As we mentioned earlier, it is possible to pick any point in $[0,1]$ and as well the numerical solutions and all their numerical derivatives up to order two will be applicable. Next, the numerical values of the absolute errors for the first and the second derivatives of the numerical solutions of Example 18 have been plotted in Figures 1 and 2, respectively, at various $x$ in $[0,1]$. As the plots show, while the value of $x$ approaches to the boundary of $[0,1]$, the numerical values for both derivatives approach smoothly to the $x$-axis. It is observed that the increase in the number of node results in a reduction in the absolute errors and correspondingly an improvement in the accuracy of the obtained solutions. This goes in agreement with the known fact, the error is decreasing, where more accurate solutions are achieved using an increase in the number of nodes. On the other hand, the cost to be paid while going in this direction is the rapid increase in the number of iterations required for convergence.

\section{Conclusions}

The applications of the RKHS algorithm were extended successfully for solving nonlinear systems of singular periodic BVPs. In this approach, reproducing kernel spaces are constructed, in which the given periodic boundary conditions of the systems can be involved. The analytical-numerical solutions were calculated in the form of a convergent series 
TABLE 1: Numerical results of the first dependent variable $u_{1}(x)$ for Example 18 at various $x$.

\begin{tabular}{lcccc}
\hline Node & Analytical solution & Numerical solution & Absolute error & Relative error \\
\hline 0.16 & 0.01806336 & 0.018061625276524 & $1.73472348 \times 10^{-6}$ & $9.60354815 \times 10^{-5}$ \\
0.32 & 0.04734976 & 0.047349379176377 & $3.80823623 \times 10^{-7}$ & $8.04277832 \times 10^{-6}$ \\
0.48 & 0.06230016 & 0.062299488510786 & $6.71489214 \times 10^{-7}$ & $1.07782904 \times 10^{-5}$ \\
0.64 & 0.05308416 & 0.053083307200229 & $8.52799771 \times 10^{-7}$ & $1.60650516 \times 10^{-5}$ \\
0.80 & 0.02560000 & 0.025599401748663 & $5.98251337 \times 10^{-7}$ & $2.33691929 \times 10^{-5}$ \\
0.96 & 0.00147456 & 0.001474456537048 & $1.03462952 \times 10^{-7}$ & $7.01653046 \times 10^{-5}$ \\
\hline
\end{tabular}

TABLE 2: Numerical results of the second dependent variable $u_{2}(x)$ for Example 18 at various $x$.

\begin{tabular}{lllll}
\hline Node & Analytical solution & Numerical solution & Absolute error & Relative error \\
\hline 0.16 & 0.990981907024073 & 0.990981358268457 & $5.48755616 \times 10^{-7}$ & $5.53749380 \times 10^{-7}$ \\
0.32 & 0.976418389339894 & 0.976418075655312 & $3.13684582 \times 10^{-7}$ & $3.21260420 \times 10^{-7}$ \\
0.48 & 0.969011305778715 & 0.969010889445081 & $4.16333634 \times 10^{-7}$ & $4.29647860 \times 10^{-7}$ \\
0.64 & 0.973575126105082 & 0.973574808804160 & $3.17300922 \times 10^{-7}$ & $3.25913135 \times 10^{-7}$ \\
0.80 & 0.987227283375627 & 0.987226574693002 & $7.08682625 \times 10^{-7}$ & $7.17851539 \times 10^{-7}$ \\
0.96 & 0.999262810592514 & 0.999262122485934 & $6.88106580 \times 10^{-7}$ & $6.88614219 \times 10^{-7}$ \\
\hline
\end{tabular}

TABLE 3: Numerical results of the first dependent variable $u_{1}(x)$ for Example 19 at various $x$.

\begin{tabular}{lcccc}
\hline Node & Analytical solution & Numerical solution & Absolute error & Relative error \\
\hline 0.16 & 0.091392 & 0.0913914732084920 & $5.26791508 \times 10^{-7}$ & $5.76408775 \times 10^{-6}$ \\
0.32 & 0.078336 & 0.0783359532733390 & $4.67266612 \times 10^{-8}$ & $5.96490263 \times 10^{-7}$ \\
0.48 & 0.009984 & 0.0099839814790730 & $1.85209270 \times 10^{-8}$ & $1.85506079 \times 10^{-6}$ \\
0.64 & -0.064512 & -0.064512278090456 & $2.78090456 \times 10^{-7}$ & $4.31067796 \times 10^{-6}$ \\
0.80 & -0.096000 & -0.096000768338354 & $7.68338354 \times 10^{-7}$ & $8.00352452 \times 10^{-6}$ \\
0.96 & -0.035328 & -0.035328843746913 & $8.43746913 \times 10^{-7}$ & $2.38832346 \times 10^{-5}$ \\
\hline
\end{tabular}

TABLE 4: Numerical results of the second dependent variable $u_{2}(x)$ for Example 19 at various $x$.

\begin{tabular}{lllll}
\hline Node & Analytical solution & Numerical solution & Absolute error & Relative error \\
\hline 0.16 & 0.017902155877180 & 0.017902128978676 & $2.68985039 \times 10^{-8}$ & $1.50252875 \times 10^{-6}$ \\
0.32 & 0.046262935319853 & 0.046262887673081 & $4.76467724 \times 10^{-8}$ & $1.02991244 \times 10^{-6}$ \\
0.48 & 0.060436519420406 & 0.060435812977117 & $7.06443289 \times 10^{-7}$ & $1.16890135 \times 10^{-5}$ \\
0.64 & 0.051723153984674 & 0.051723041237000 & $1.12747674 \times 10^{-7}$ & $2.17982982 \times 10^{-6}$ \\
0.80 & 0.025277807184269 & 0.025277410907301 & $3.96276968 \times 10^{-7}$ & $1.56768728 \times 10^{-5}$ \\
0.96 & 0.001473473903948 & 0.001472806316284 & $6.67587664 \times 10^{-7}$ & $4.53070571 \times 10^{-4}$ \\
\hline
\end{tabular}

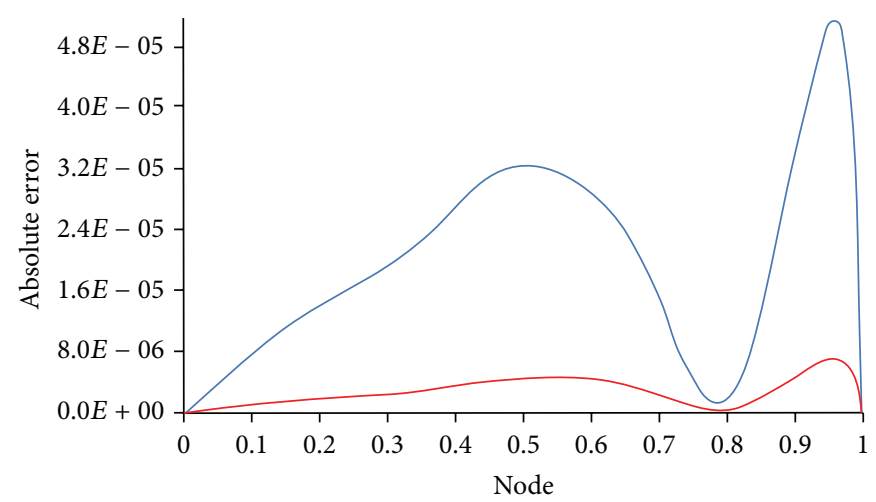

FIGURE 2: The numerical values of the absolute error function for the second derivative of Example 18: blue: the first dependent variable and red: the second dependent variable. 
in the space $W_{2}^{3}[0,1]$ with easily computable components; in the meantime the $n$-term numerical solutions are obtained and are proved to converge to the analytical solutions. The solution methodology is based on generating the orthogonal basis from the obtained kernel functions; whilst the orthonormal basis is constructing in order to formulate and utilize the solutions with series form in the space $W_{2}^{3}[0,1]$. Further, an error estimation and error bound based on the reproducing kernel theory are proposed in order to capture the behavior of the numerical solutions. Tabulated data, graphical results, and numerical comparisons with the analytical solutions are presented and discussed quantitatively to illustrate the numerical solutions. The basic ideas of this iterative novel approach can be widely employed to solve other strongly nonlinear singular systems.

\section{Appendices}

Here, we provide a detailed proof of Theorem 3 and the expansion formulas for the unknown coefficients $a_{i}(x)$ and $b_{i}(x), i=1,2, \ldots, 6$, of the reproducing kernel function $R_{x}(y)$ in the space $W_{2}^{3}[0,1]$.

\section{A. Proof of Theorem 3}

The proof of the completeness and reproducing property of $W_{2}^{3}[0,1]$ is similar to the proof in [21]; let us find out the expression form of $R_{x}(y)$. Through several integrations by parts, we obtain

$$
\begin{aligned}
& \int_{0}^{1} u^{\prime \prime \prime}(y) \partial_{y}^{3} R_{x}(y) d y \\
& =\left.\sum_{i=0}^{2}(-1)^{i} u^{(i)}(y) \partial_{y}^{5-i} R_{x}(y)\right|_{y=0} ^{y=1} \\
& \quad-\int_{0}^{1} u(y) \partial_{y}^{6} R_{x}(y) d y
\end{aligned}
$$

Thus, from (3) one can write

$$
\begin{aligned}
& \left\langle u(y), R_{x}(y)\right\rangle_{W_{2}^{3}} \\
& =\sum_{i=0}^{2} u^{(i)}(0)\left[\partial_{y}^{i} R_{x}(0)+(-1)^{i+1} \partial_{y}^{5-i} R_{x}(0)\right] \\
& \quad+\sum_{i=0}^{2}(-1)^{i} u^{(i)}(1) \partial_{y}^{5-i} R_{x}(1) \\
& \quad-\int_{0}^{1} u(y) \partial_{y}^{6} R_{x}(y) d y .
\end{aligned}
$$

Since $R_{x}(y) \in W_{2}^{3}[0,1]$, it follows that $R_{x}(0)=R_{x}(1)$ and $\partial_{y}^{1} R_{x}(0)=\partial_{y}^{1} R_{x}(1)$. Again, since $u(x) \in W_{2}^{3}[0,1]$, it yields that $u^{(i)}(a)=u^{(i)}(b), i=0,1$. Hence,

$$
\begin{aligned}
& \left\langle u(y), R_{x}(y)\right\rangle_{W_{2}^{3}} \\
& =\sum_{i=0}^{2} u^{(i)}(0)\left[\partial_{y}^{i} R_{x}(0)+(-1)^{i+1} \partial_{y}^{5-i} R_{x}(0)\right] \\
& \quad+\sum_{i=0}^{2}(-1)^{i} u^{(i)}(1) \partial_{y}^{5-i} R_{x}(1) \\
& \quad-\int_{0}^{1} u(y) \partial_{y}^{6} R_{x}(y) d y+c_{1}(u(0)-u(1)) \\
& \quad+c_{2}\left(u^{\prime}(0)-u^{\prime}(1)\right) .
\end{aligned}
$$

On the other hand, if $\partial_{y}^{3} R_{x}(1)=0, R_{x}(0)-\partial_{y}^{5} R_{x}(0)+c_{1}=$ $0, \partial_{y}^{2} R_{x}(0)-\partial_{y}^{3} R_{x}(0)=0, \partial_{y}^{5} R_{x}(1)-c_{1}=0, \partial_{y}^{1} R_{x}(0)+$ $\partial_{y}^{4} R_{x}(0)+c_{2}=0$, and $\partial_{y}^{4} R_{x}(1)+c_{2}=0$, then (A.3) implies that $\left\langle u(y), R_{x}(y)\right\rangle_{W_{2}^{3}}=\int_{0}^{1} u(y)\left(-\partial_{y}^{6} R_{x}(y)\right) d y$. Now, for any $x \in[0,1]$, if $R_{x}(y)$ satisfies

$$
\partial_{y}^{6} R_{x}(y)=-\delta(x-y), \quad \delta \text { dirac-delta function, }
$$

then $\left\langle u(y), R_{x}(y)\right\rangle_{W_{2}^{3}}=u(x)$. Obviously, $R_{x}(y)$ is the reproducing kernel function of $W_{2}^{3}[0,1]$. Next, we give the expression form of $R_{x}(y)$. The auxiliary formula of (A.4) is given by $\lambda^{6}=0$, and its auxiliary values are $\lambda=0$ with multiplicity 6 . So, let the expression form of $R_{x}(y)$ be as defined in (4). But on the other aspect as well, for (A.4), let $R_{x}(y)$ satisfy the equation $\partial_{y}^{m} R_{x}(x+0)=\partial_{y}^{m} R_{x}(x-0)$, $m=0,1,2,3$, 4. Integrating $\partial_{y}^{6} R_{x}(y)=-\delta(x-y)$ from $x-\varepsilon$ to $x+\varepsilon$ with respect to $y$ and letting $\varepsilon \rightarrow 0$, we have the jump degree of $\partial_{y}^{5} R_{x}(y)$ at $y=x$ given by $\partial_{y}^{5} R_{x}(x+0)-\partial_{y}^{5} R_{x}(x-0)=$ -1 . Through the last descriptions the unknown coefficients $a_{i}(x)$ and $b_{i}(x), i=1,2, \ldots, 6$, of (4) can be obtained. This completes the proof.

\section{B. Coefficients of the Reproducing Kernel Function $R_{x}(y)$}

$$
\begin{aligned}
& a_{1}(x)=1, \\
& a_{2}(x)=\frac{1}{3867} x\left(27-60 x-20 x^{2}+85 x^{3}-32 x^{4}\right), \\
& a_{3}(x)=-\frac{1}{15468} x\left(240-963 x+968 x^{2}-247 x^{3}\right. \\
& \left.\quad+2 x^{4}\right), \\
& a_{4}(x)=-\frac{1}{46404} x\left(240-963 x+968 x^{2}-247 x^{3}\right. \\
& \left.\quad+2 x^{4}\right)
\end{aligned}
$$




$$
\begin{aligned}
& a_{5}(x)=\frac{1}{9208} x\left(-1827+1482 x+494 x^{2}-166 x^{3}\right. \\
& \left.+17 x^{4}\right) \\
& a_{6}(x)=\frac{1}{464040}\left(3867-3840 x-60 x^{2}-20 x^{3}\right. \\
& \left.\quad+85 x^{4}-32 x^{5}\right) \\
& b_{1}(x)=1+\frac{1}{120} x^{5} \\
& b_{2}(x)=\frac{1}{30936} x\left(216-480 x-160 x^{2}-609 x^{3}\right. \\
& \left.\quad-256 x^{4}\right), \\
& \quad(x)=-\frac{1}{15468} x\left(240-963 x-321 x^{2}-247 x^{3}\right. \\
& \left.\quad+2 x^{4}\right) \\
& \left.\quad+32 x^{4}\right) \\
& b_{6}(x)=-\frac{1}{464040} x\left(3840+60 x+20 x^{2}-85 x^{3}\right. \\
& b_{4}(x)=-\frac{1}{46404} x\left(240+2904 x+968 x^{2}-247 x^{3}\right. \\
& b_{5}(x)=\frac{1}{92808} x\left(2040+1482 x+494 x^{2}-166 x^{3}\right. \\
& +2
\end{aligned}
$$

[3] G. Kitzhofer, O. Koch, P. Lima, and E. Weinmüller, "Efficient numerical solution of the density profile equation in hydrodynamics," Journal of Scientific Computing, vol. 32, no. 3, pp. 411424, 2007.

[4] P. Lima, N. Chemetov, N. Konyukhova, and A. Sukov, "Analytical-numerical approach to a singular boundary value problem," in Proceedings of 24th CILAMCE, Ouro Preto, Brazil.

[5] I. Rachůnkov, O. Koch, G. Pulverer, and E. Weinmüller, "On a singular boundary value problem arising in the theory of shallow membrane caps," Journal of Mathematical Analysis and Applications, vol. 332, no. 1, pp. 523-541, 2007.

[6] O. Koch and E. Weinmüller, "Analytical and numerical treatment of a singular initial value problem in avalanche modeling," Applied Mathematics and Computation, vol. 148, no. 2, pp. 561570, 2004.

[7] D. M. McClung and A. I. Mears, "Dry-flowing avalanche run-up and run-out," Journal of Glaciology, vol. 41, no. 138, pp. 359-372, 1995.

[8] U. M. Ascher, R. M. Mattheij, and R. D. Russell, Numerical Solution of Boundary Value Problems for Ordinary Differential Equations, vol. 13 of Classics in Applied Mathematics, SIAM, Philadelphia, Pa, USA, 1995.

[9] O. Abu Arqub, Z. Abo-Hammour, S. Momani, and N. Shawagfeh, "Solving singular two-point boundary value problems using continuous genetic algorithm," Abstract and Applied Analysis, vol. 2012, Article ID 205391, 25 pages, 2012.

[10] Z. Abo-Hammour, O. Abu Arqub, O. Alsmadi, S. Momani, and A. Alsaedi, "An optimization algorithm for solving systems of singular boundary value problems," Applied Mathematics \& Information Sciences, vol. 8, no. 6, pp. 2809-2821, 2014.

[11] F. Zhu, L. Liu, and Y. Wu, "Positive solutions for systems of a nonlinear fourth-order singular semipositone boundary value problems," Applied Mathematics and Computation, vol. 216, no. 2, pp. 448-457, 2010.

[12] W. Liu, L. Liu, and Y. Wu, "Positive solutions of a singular boundary value problem for systems of second-order differential equations," Applied Mathematics and Computation, vol. 208, no. 2, pp. 511-519, 2009.

\section{Conflict of Interests}

The author declares that there is no conflict of interests regarding the publication of this paper.

\section{Acknowledgment}

The author would like to express his gratitude to the unknown referees for carefully reading the paper and their helpful comments.

\section{References}

[1] C. J. Budd, S. Chen, and R. D. Russell, "New self-similar solutions of the nonlinear Schrödinger equation with moving mesh computations," Journal of Computational Physics, vol. 152, no. 2, pp. 756-789, 1999.

[2] C. Budd, O. Koch, and E. Weinmüller, "From nonlinear PDEs to singular ODEs," Applied Numerical Mathematics, vol. 56, no. 3-4, pp. 413-422, 2006.

13] W.-S. Cheung and P. J. Wong, "Fixed-sign solutions for a system of singular focal boundary value problems," Journal of Mathematical Analysis and Applications, vol. 329, no. 2, pp. 851869, 2007.

[14] Z. Wei, "Positive solution of singular Dirichlet boundary value problems for second order differential equation system," Journal of Mathematical Analysis and Applications, vol. 328, no. 2, pp. 1255-1267, 2007.

[15] B. Liu, L. Liu, and Y. Wu, "Positive solutions for singular systems of three-point boundary value problems," Computers \& Mathematics with Applications, vol. 53, no. 9, pp. 1429-1438, 2007.

[16] M. Cui and Y. Lin, Nonlinear Numerical Analysis in the Reproducing Kernel Space, Nova Science, New York, NY, USA, 2009.

[17] A. Berlinet and C. Thomas-Agnan, Reproducing Kernel Hilbert Spaces in Probability and Statistics, Kluwer Academic Publishers, Boston, Mass, USA, 2004.

[18] A. Daniel, Reproducing Kernel Spaces and Applications, Springer, Basel, Switzerland, 2003.

[19] H. L. Weinert, Reproducing Kernel Hilbert Spaces: Applications in Statistical Signal Processing, Hutchinson Ross, 1982.

[20] Y.Z. Lin, M. G. Cui, and L. H. Yang, "Representation of the exact solution for a kind of nonlinear partial differential equation," Applied Mathematics Letters, vol. 19, no. 8, pp. 808-813, 2006. 
[21] L.-H. Yang and Y. Lin, "Reproducing kernel methods for solving linear initial-boundary-value problems," Electronic Journal of Differential Equations, vol. 2008, no. 29, 11 pages, 2008.

[22] O. Abu Arqub and M. Al-Smadi, "Numerical algorithm for solving two-point, second-order periodic boundary value problems for mixed integro-differential equations," Applied Mathematics and Computation, vol. 243, pp. 911-922, 2014.

[23] S. Momani, O. Abu Arqub, T. Hayat, and H. Al-Sulami, "A computational method for solving periodic boundary value problems for integro-differential equations of Fredholm-Volterra type," Applied Mathematics and Computation, vol. 240, pp. 229239, 2014

[24] M. Al-Smadi, O. Abu Arqub, and S. Momani, "A computational method for two-point boundary value problems of fourth-order mixed integrodifferential equations," Mathematical Problems in Engineering, vol. 2013, Article ID 832074, 10 pages, 2013.

[25] O. Abu Arqub, M. Al-Smadi, and S. Momani, "Application of reproducing kernel method for solving nonlinear FredholmVolterra integrodifferential equations," Abstract and Applied Analysis, vol. 2012, Article ID 839836, 16 pages, 2012.

[26] O. A. Arqub, M. Al-Smadi, and N. Shawagfeh, "Solving Fredholm integro-differential equations using reproducing kernel Hilbert space method," Applied Mathematics and Computation, vol. 219, no. 17, pp. 8938-8948, 2013.

[27] N. Shawagfeh, O. Abu Arqub, and S. Momani, "Analytical solution of nonlinear second-order periodic boundary value problem using reproducing kernel method," Journal of Computational Analysis and Applications, vol. 16, no. 4, pp. 750-762, 2014.

[28] M. Al-Smadi, O. Abu Arqub, and A. El-Ajou, "A numerical iterative method for solving systems of first-order periodic boundary value problems," Journal of Applied Mathematics, vol. 2014, Article ID 135465, 10 pages, 2014.

[29] O. Abu Arqub, "An iterative method for solving fourth-order boundary value problems of mixed type integro-differential equations," Journal of Computational Analysis and Applications, vol. 8, pp. 857-874, 2015.

[30] B. Maayah, S. Bushnaq, S. Momani, and O. Abu Arqub, "Iterative multistep reproducing kernel Hilbert space method for solving strongly nonlinear oscillators," Advances in Mathematical Physics, vol. 2014, Article ID 758195, 7 pages, 2014.

[31] O. Abu Arqub, M. AL-Smadi, S. Momani, and T. Hayat, "Numerical solutions of fuzzy differential equations using reproducing kernel Hilbert space method," Soft Computing, In press.

[32] F. Geng and M. Cui, "Solving singular nonlinear second-order periodic boundary value problems in the reproducing kernel space," Applied Mathematics and Computation, vol. 192, no. 2, pp. 389-398, 2007.

[33] C.-I. Li and M.-G. Cui, "The exact solution for solving a class nonlinear operator equations in the reproducing kernel space," Applied Mathematics and Computation, vol. 143, no. 2-3, pp. 393-399, 2003.

[34] W. Jiang and Z. Chen, "Solving a system of linear Volterra integral equations using the new reproducing kernel method," Applied Mathematics and Computation, vol. 219, no. 20, pp. 10225-10230, 2013.

[35] F. Geng and M. Cui, "A reproducing kernel method for solving nonlocal fractional boundary value problems," Applied Mathematics Letters, vol. 25, no. 5, pp. 818-823, 2012.

[36] F. Z. Geng and S. P. Qian, "Reproducing kernel method for singularly perturbed turning point problems having twin boundary layers," Applied Mathematics Letters, vol. 26, no. 10, pp. 998-1004, 2013.

[37] W. Jiang and Z. Chen, "A collocation method based on reproducing kernel for a modified anomalous subdiffusion equation," Numerical Methods for Partial Differential Equations, vol. 30, no. 1, pp. 289-300, 2014.

[38] F. Z. Geng, S. P. Qian, and S. Li, "A numerical method for singularly perturbed turning point problems with an interior layer," Journal of Computational and Applied Mathematics, vol. 255, pp. 97-105, 2014. 


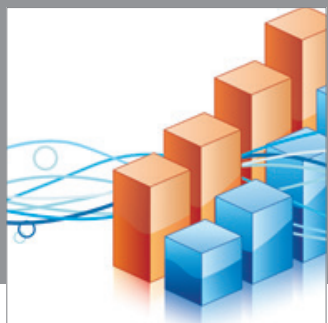

Advances in

Operations Research

mansans

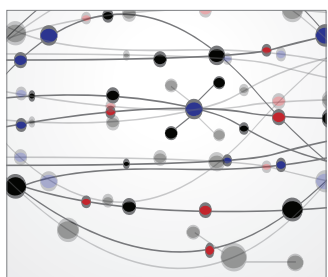

The Scientific World Journal
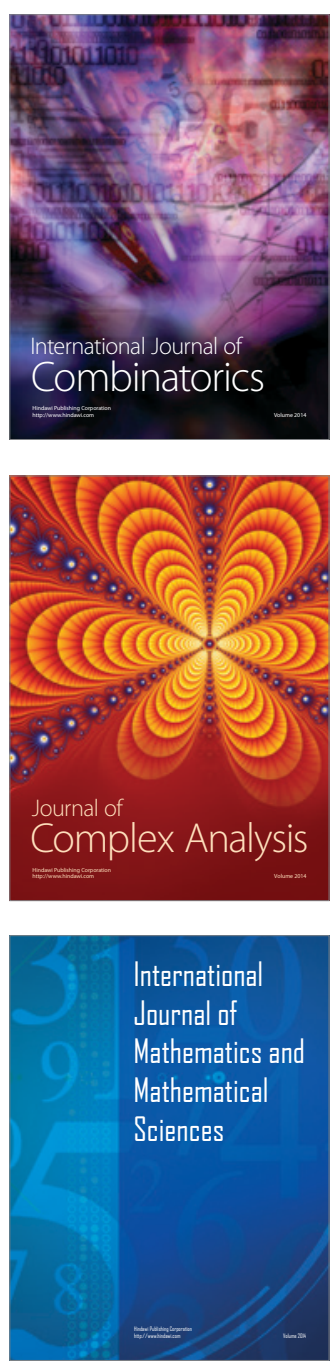
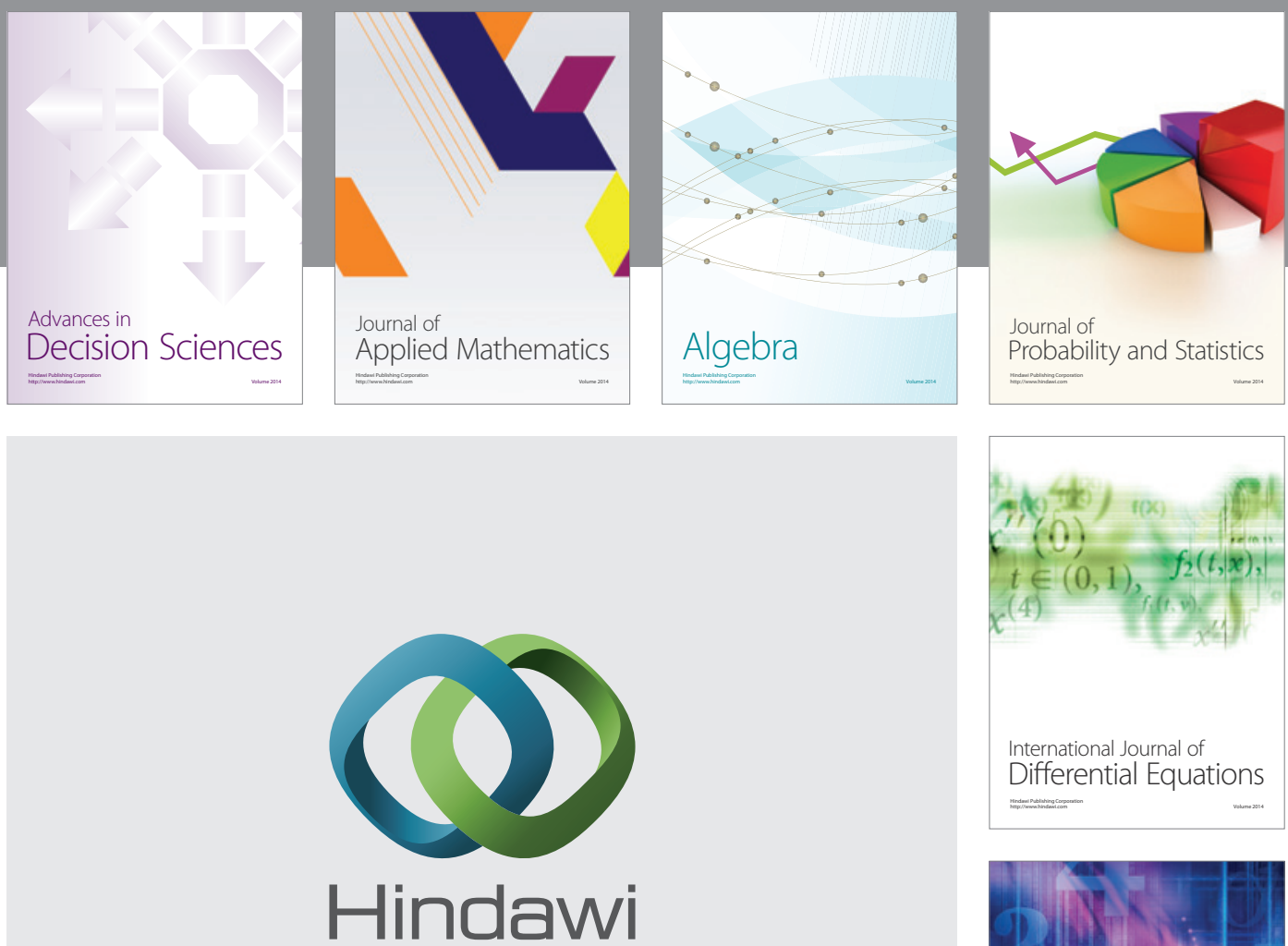

Submit your manuscripts at http://www.hindawi.com
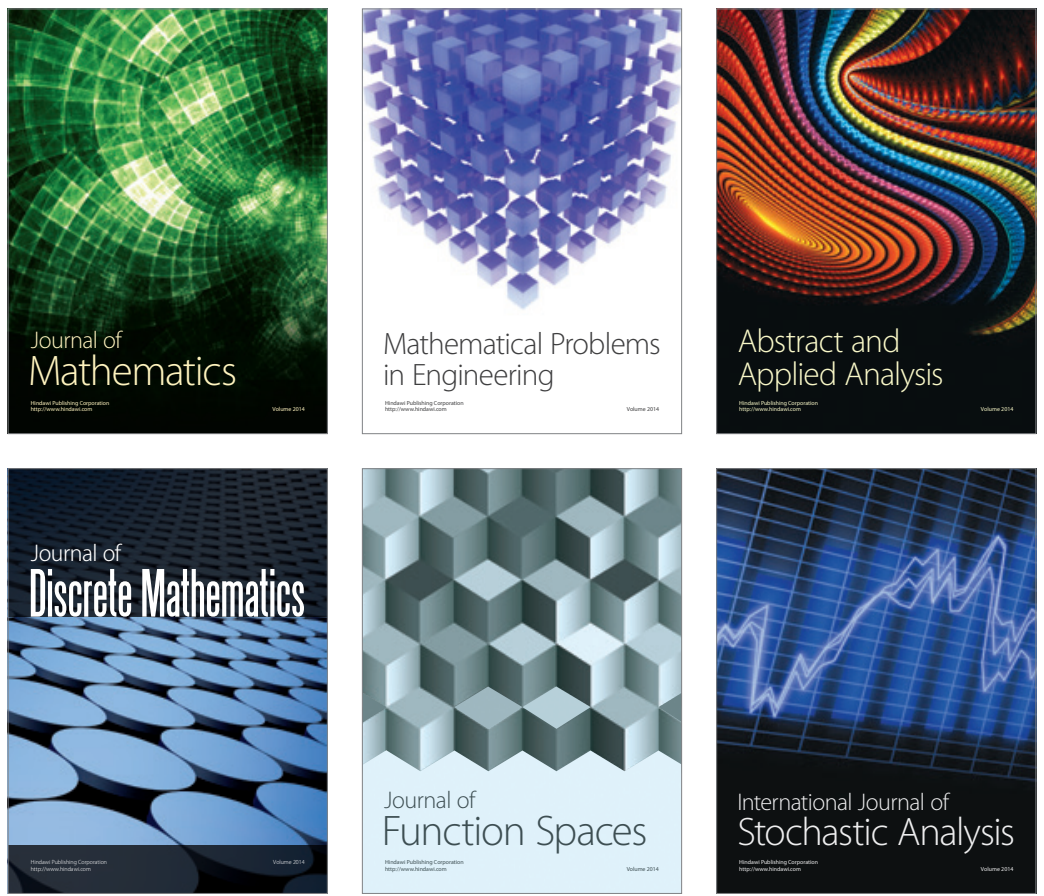

Journal of

Function Spaces

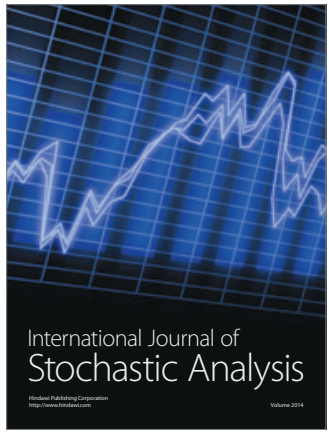

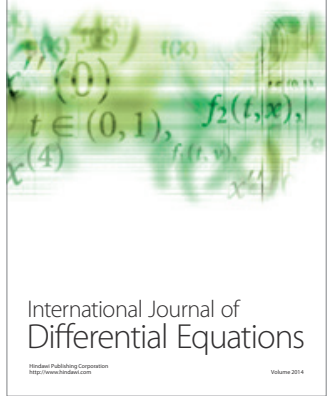
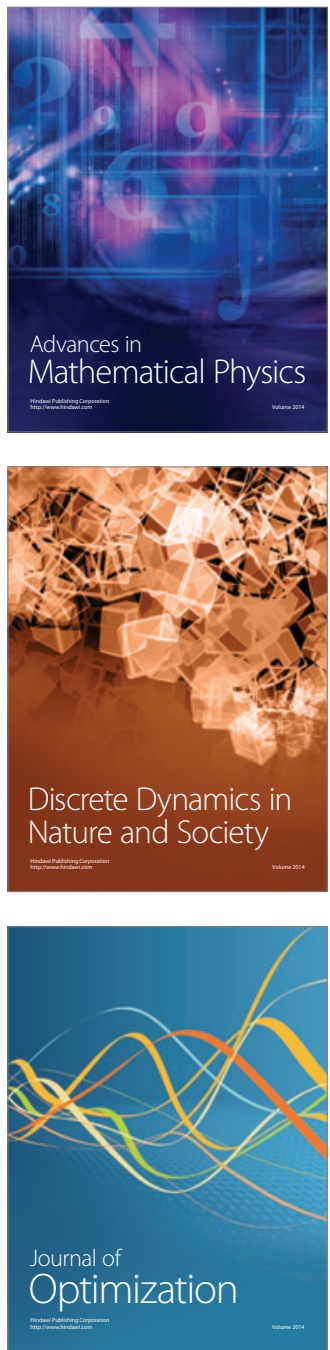